\title{
Assessment of the therapeutic accuracy of cone beam computed tomography-guided nasopharyngeal carcinoma radiotherapy
}

\author{
JIABIN LIU ${ }^{1,2^{*}}$, KHUMBULA MAITIREAZVO LYMAN $^{1 *}$, ZHENHUA DING $^{1}$ and LIANG ZHOU ${ }^{1}$ \\ ${ }^{1}$ Department of Radiation Medicine, Guangdong Provincial Key Laboratory of Tropical Disease Research, \\ School of Public Health, Southern Medical University; ${ }^{2}$ Department of Radiation Oncology, \\ Nanfang Hospital, Southern Medical University, Guangzhou, Guangdong 510515, P.R. China
}

Received May 20, 2018; Accepted April 8, 2019

DOI: $10.3892 / \mathrm{ol} .2019 .10412$

\begin{abstract}
The aim of the present study was to determine the ability of cone beam computed tomography (CBCT) to improve the accuracy of nasopharyngeal carcinoma (NPC) radiotherapy by analyzing the setup and inter-fraction errors at different levels and directions of the target volumes. A total of 113 patients with NPC who were undergoing intensity-modulated radiotherapy were recruited for the present study. Each patient had at least three CBCT exams prior to the start of radiation therapy. Three anatomic bony landmarks, including the upper neck, lower neck and head, were used to represent the different levels of assessment. The positioning errors were registered in three planes throughout the course of radiotherapy: The right-left (RL), superior-inferior (SI) and anterior-posterior (AP) directions. The planning CT images were matched with the CBCT images to determine the naso-pharynx shifts. A receiver operating characteristic curve was plotted to establish the specificity and sensitivity of CBCT. The planning target volume margin (MPTV) for the head was $0.9 \mathrm{~mm}, 1.4 \mathrm{~mm}$ for the upper neck and $2.0 \mathrm{~mm}$ for the lower neck. MPTVs of 1.5, 0.6 and $2.2 \mathrm{~mm}$ in the RL, SI and AP directions, respectively, were detected. In addition, there was evidence of setup errors in the three planes (RL, SI and AP) with the greatest error observed in the AP direction. Furthermore, the setup uncertainties in the neck region were greater than those of the head. In conclusion, CBCT could greatly improve the accuracy of radiotherapy by minimizing the setup errors and MPTV.
\end{abstract}

Correspondence to: Professor Liang Zhou, Department of Radiation Medicine, Guangdong Provincial Key Laboratory of Tropical Disease Research, School of Public Health, Southern Medical University, 1838 Northern Guangzhou Avenue, Guangzhou, Guangdong 510515, P.R. China

E-mail: zhzliang@smu.edu.cn

${ }^{*}$ Contributed equally

Key words: cone beam computed tomography, intensity-modulated radiation therapy, nasopharyngeal carcinoma, setup error, planning target volume margin, receiver operating characteristic analysis

\section{Introduction}

Nasopharyngeal carcinoma (NPC) is highly prevalent in southern China and regions of southeast Asia, and possesses great metastatic potential (1). The majority of patients with NPC are diagnosed with advanced stage tumors (stages III or IV) (1). Risk factors including environmental carcinogens, genetic alternations and Epstein-Barr virus infections are thought to be responsible for the pathogenesis of NPC (2). Radiotherapy (RT) is the primary and only curative treatment for NPC (1). In modern radiation oncology centers, in order to maximize tumor control and keep organs at risk (OAR) irradiation at a minimum, intensity-modulated RT (IMRT) is the preferred treatment of nasopharyngeal neoplasms. IMRT was designed to deliver tumoricidal doses of ionizing radiation to the tumor while minimizing the doses received by adjacent normal tissues, thereby improving patient quality of life due to the higher local control and lower toxicity levels (3). However, although IMRT is associated with numerous benefits, anatomical changes and geometrical alterations throughout the course of RT have restricted its performance (4). Tumor shrinkage, weight loss and soft tissue alterations have all been reported as significant causative processes of anatomical changes throughout the treatment of patients with NPC (5). In addition, positional errors may be due to differences between the equipment in the simulation and treatment rooms, and immobilization devices (3). Therefore, a method of reducing the significant sources of uncertainty during IMRT treatment is required.

Advances in 3D imaging technology has generated a number of different types of kilovoltage $(\mathrm{kV})$ or megavoltage imaging systems on linear accelerators, among which cone beam computed tomography (CBCT) has gained widespread utilization for resolving the poor accuracy of IMRT by ultimately minimizing the systemic and random errors through offline analysis and online correction (6,7). Although a fixed planning target volume margin (MPTV) is usually applied for the all of the regions of interest (ROI) in CBCT, anatomic structural changes vary during the treatment course, which may not generate or cause equal setup errors, particularly when there is therapeutic movement from the head to the neck during the course of NPC treatment $(6,7)$. Therefore, it is important to make adjustments during treatment according 
to the actual measurements in CBCT in order to ensure the success of RT.

RT setup errors are the differences between the intended and actual position of the patient. Ordinarily, the errors are classified into random, systematic inter-fractional errors (i.e. deviancy between different fractions). Systematic error is a deviation that occurs in the same direction and is of a similar size for each fraction throughout the course of treatment. It is calculated as the standard deviation (SD) of the distribution of mean errors for each individual patient. These errors can be introduced into the patient's treatment at the localization, planning or treatment delivery stages; these are at times referred to as treatment preparation errors (8). On the other hand, random error is a deviation that can vary in direction and magnitude for each delivered treatment fraction. They are usually introduced or occur at the treatment delivery stage and are therefore referred to as treatment (daily) execution errors (9).

The present study investigated the setup errors by analyzing the Planning Target Volume (PTV) and MPTV of the right-left (RL), superior-inferior (SI) and anterior-posterior (AP) directions of the upper neck, lower neck and head levels from the CBCT data of 113 patients with NPC receiving IMRT treatment. The PTV is the volume that allows for uncertainties in the delivery and planning stage, it is the one that ensures adequate treatment delivery to the ROI and can in some instances extend outside the patient. By systemically analyzing the results, it was expected that the major sources of setup errors would be identified, in order to establish a unified standard for future CBCT practical operations.

\section{Materials and methods}

Patient information. Initially, 120 patients were recruited to the present study regardless of the stage of NPC at Department of Radiation Oncology, Nanfang Hospital (Guangzhou, China) between January 2016 and December 2017. The inclusion criterion was to include patients who had undergone at least three CBCT scans prior to the commencement of RT, thus 7 patients were subsequently removed from the study as they had received only two CBCT scans during the data collection phase. The total dose of radiotherapy was divided into multiple treatments, so-called split-dose treatments. For a complete radiotherapy, at least three occasions of split-dose treatments were performed with one week of interval. A one time CBCT scan was performed for tumor localization and correction of patient's body position for maximizing the benefit of radiotherapy $5 \mathrm{~min}$ prior to each split-dose treatment.

The present study was approved by the Institutional Review Board of Nanfang Hospital (Guangzhou, China), and all patients provided written informed consent for the use of their imaging data. Staging was done using the 2008 nasopharyngeal carcinoma staging system in People's Republic of China (10). A summary of the patients' information is presented in Table I.

Procedure of conventional CT. Patients were laid down in the supine position and were immobilized using the Klarity head and shoulder thermoplastic immobilization system whilst the images were acquired using the Philips Brilliance Big Bore CT simulator (Philips Healthcare) (1.0 mm slice thick). The scan covered the regions from the vertex of the head to the manubriosternal joint. Through the use of the Digital Imaging and Communications in Medicine network (11), datasets from the planning CT were transferred to the Varian Eclipse treatment planning system (v10.0; Varian Medical Systems, Inc.). Axial slices of the planning CT scan were used as contours for the target delineation of the patients included in the present study. The high-risk regions surrounding the primary tumor were clinical target volume 1 (CTV1), as were all of the neck nodes at high risk, while the low-risk node region below the CTV1 was CTV2. The PTVs and planning OAR volumes were determined by adding a 3-mm margin to the respective CTVs and the corresponding structures including the bilateral parotids, spinal cord and brainstem. The primary (planning) CT scan was used as the control image.

Procedure of CBCT imaging. Images were captured following the conventional alignment of the in-room lasers with markings on the thermoplastic masks. VARIAN On-board Imaging system (Varian Medical Systems, Inc.) was used to obtain the Pretreatment kV CBCT scans, employing the exposure parameters presented in Table II; the reconstruction slice thickness was $1 \mathrm{~mm}$. Prior to the RT session, each patient underwent a $\mathrm{kV}$ CBCT scan. In any instance where the translational setup error was $>3 \mathrm{~mm}$ in any direction a setup correction was performed. The deformation of the images that occurred in the co-registration process was also taken into account.

All of the acquired images were analyzed online using VARIAN On-board Imaging Systems software (v10.0; Varian Medical Systems, Inc.). The CBCT scan was matched to the planning CT scan via automatic bone matching using a combination of automatic registration and the manual fine-tuning method for image registration. Bony landmarks were used to represent the different regions: The nasal septum and pterygoid process represented the head; the upper neck was represented by cervical vertebrae 1-3 and the lower neck was denoted by cervical vertebrae 4-6 (12). Comparisons were made between the setup errors of the $\mathrm{kV} \mathrm{CBCT}$ image and the CT image registration recorded in the different regions, and the dimensions were also recorded in three directions (RL, SI and AP). Following this, comparisons of the differences among the three regions (upper neck, lower neck and head) in the acquired registration images were made.

Statistical analysis. The data were analyzed using Microsoft Excel 2016 (Microsoft Corporation). Based on the Stroom definition of the error estimation method (13), the mean value of each patient's position error was an individual systemic error, and the SD of each patient's position error was the individual random error. The group systematic errors were the SDs of the individual systematic errors, and the random errors were the SDs of the individual random errors. Setup errors are expressed as the systematic errors \pm random errors. One-way analysis of variance (ANOVA) was performed to analyze the differences in setup errors in the upper neck, lower neck and head prior to corrections or treatment. ANOVA or Kruskal-Wallis H-test were employed for multiple comparisons among the upper neck, lower neck and head regions, and the post hoc Duncan test was performed. $\mathrm{P}<0.05$ was considered to indicate a statistically significant difference. The classical van-Herk 
Table I. Summary of patient information.

\begin{tabular}{lcc}
\hline Characteristics & Median (range) & Cases, $\mathrm{n}$ \\
\hline Age, years & $49(23-75)$ & \\
Sex & \\
Male & 88 \\
Female & 25 \\
Clinical stage & \\
I & 8 \\
II & 27 \\
III & 43 \\
IV & 35 \\
\hline
\end{tabular}

Table II. Pretreatment kV CBCT parameters.

\begin{tabular}{lc}
\hline Parameter & Setting \\
\hline Tube voltage, kilovolt & 100 \\
Tube current, milliampere & 10 \\
Pulse duration, milliampere second & 10 \\
Approximate frames, $\mathrm{n}$ & 361 \\
Total angle, $^{\circ}$ & 200 \\
Scan field of view, mm $^{\mathrm{a}}$ & $180 \times 140$ \\
Image reconstruction matrix, pixels & $512 \times 512$ \\
\hline
\end{tabular}

apresented as the diameter $\mathrm{x}$ height.

formula, MPTV $=2.5 \Sigma+0.7 \sigma(8)$, was utilized to estimate the ideal CTV-to-MPTV, where $\Sigma$ is the systematic error and $\sigma$ is the random error.

Receiver operating characteristic (ROC) analysis. A ROC curve is a plot of the true positive rate against the false positive rate for the different possible cut-off points of a diagnostic test. Specificity and sensitivity are the measures used to determine the diagnostic accuracy of a test (14). Using SPSS (version 24; IBM Corp.), ROC curves were constructed through a comparison of the individual systematic errors with the various MPTV values at the three different levels (upper neck, lower neck and head). The combined test summarized the diagnostic accuracy of a test by means of a single number. The curve is associated with numerous advantages including the illustration of all of the cut-off points of a diagnostic test. It also reveals the associations between the sensitivity of a test and its specificity, though it is not affected by the prevalence of a condition (for example in the present study, the prevalence of setup errors) and it is possible for researchers to compute important summary measures of accuracy using these curves (14).

For example, if one wished to test if a certain sample has a condition under investigation, in the present study this being the presence of setup errors in patients undergoing RT, the case sensitivity would be the proportion of true positives (those with actual setup shifts). The specificity on the other hand is the proportion of true negatives-the proportion of the cases that had no setup errors among those that did not have any shifts.

In a ROC curve the sensitivity (true positive rate) is plotted against the false positive rate (1-Specificity) for different cut-off points. Any point on the ROC plot resembles a sensitivity corresponding to a particular decision threshold. A test that has perfect discrimination (the absence of an overlap between two distributions), will produce a curve that passes through the upper left corner (100\% sensitivity, $100 \%$ specificity). Hence, the closer the ROC plot is to the upper left corner, the greater the overall accuracy of the test (14).

\section{Results}

Systemic measurement of setup errors. In total, 4,613 position verification scans were performed and analyzed. A comparison was made between these acquired images and the corresponding planning CT scan images to determine the positional shifts and ultimately measure the shifts of the bony reference regions in the AP, SI and RL directions (Figs. 1-3; representative images from one patient). The planning CT image was used as the control for each patient.

To evaluate the setup errors, the ROI positional shifts were measured weekly, with each patient having at least three scans during the RT course. The results revealed that deviation in the head was in the range of $0-4 \mathrm{~mm}$ in the AP direction, 0-3 $\mathrm{mm}$ in the RL direction and $0-2 \mathrm{~mm}$ in the SI direction. The prevalence of errors $>2 \mathrm{~mm}$ were $7(2.1 \%)$ in the AP direction. In the upper neck, the range of deviations were 0-2, 0-3 and 0-2 mm in the RL, SI and AP directions, respectively. The frequencies of errors $>2 \mathrm{~mm}$ were $5(1.5 \%)$ in the SI direction. Bony reference deviation in the lower neck was in the range of $0-5 \mathrm{~mm}$ in the RL direction, $0-2 \mathrm{~mm}$ in the SI direction and 0-3 $\mathrm{mm}$ in the AP direction (Fig. 4). The incidence of errors $>2 \mathrm{~mm}$ in the RL, SI and AP directions were $62(18.3 \%)$ in the RL direction, 0 in the SI direction and $2(0.6 \%)$ in the AP direction (Table III).

Calculations to estimate the group systematic and random errors were conducted using the Stroom definition of error estimation (13). The results for group systematic and random errors were 0.509 and $0.208 \mathrm{~mm}$ in the RL direction, respectively; in the SI direction they were 0.227 and $0.160 \mathrm{~mm}$ for group systematic errors and random errors, respectively; and the group systematic and random errors in the AP direction were 0.755 and $0.345 \mathrm{~mm}$, respectively (Table IV).

AP direction possesses the highest MPTV following CBCT evaluation. Based on the van-Herk formula(MPTV $=2.5 \Sigma+0.7 \sigma)$, the ideal MPTVs were derived from the setup errors. With the aim to accurately deliver radiation doses to the targets and the associated surrounding normal tissues, the margins required in the different directions were $1.418,0.566$ and $2.129 \mathrm{~mm}$ in the RL, SI and AP directions, respectively (Table IV). When a comparison was made amongst the three anatomical levels there was an increase in the MPTVs from the cranial to the caudal region. With regard to different anatomic levels, the overall calculated MPTVs were the greatest in the lower neck ( $2 \mathrm{~mm}$ ) followed by the upper neck region $(1.4 \mathrm{~mm})$. At the head level, the calculated margin was $0.9 \mathrm{~mm}$, as presented in Table V. 
A

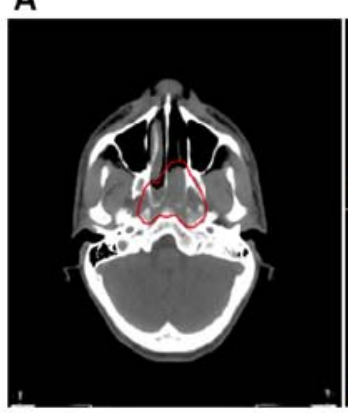

B

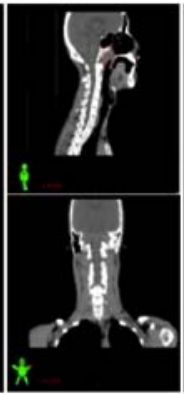

C

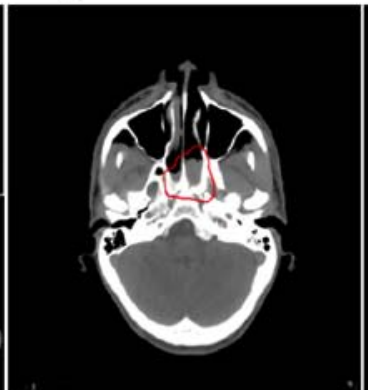

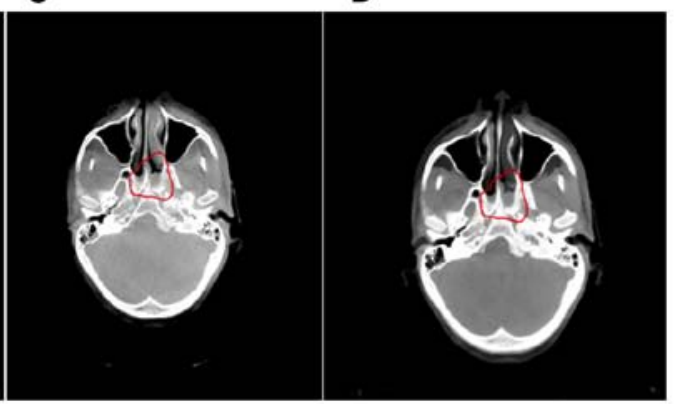

Figure 1. Image registration in the head region by automatic bone matching and manual fine-tuning. (A) Planning CT images of the cross section, coronal plane and sagittal plane in a patient with NPC; automatic bone matching and manual fine-tuning methods were performed using the nasal septum as a bony landmark. (B) Image of the head in planning CT. (C) Image of the head in cone-beam CT. (D) Typical fusion image of CBCT superimposed with planning CT image. The primary gross volume is outlined in red. CT, computed tomography; NPC, nasopharyngeal carcinoma.

A

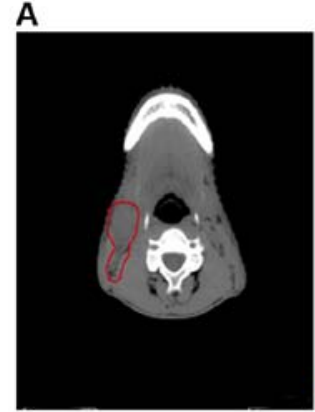

B

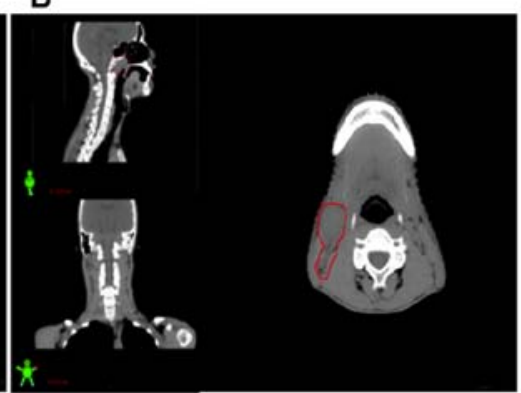

C

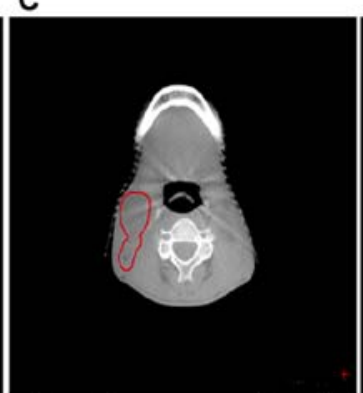

D

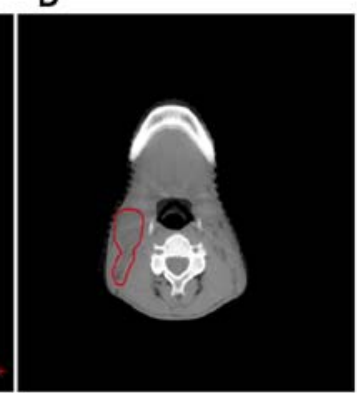

Figure 2. Image registration in the upper neck region by automatic bone matching and manual fine-tuning. (A) Planning CT images of the cross section, coronal plane and sagittal plane in a patient with NPC; cervical vertebrae 1-3 were used as a bony landmark for automatic bone matching and manual fine-tuning method. (B) Image of the upper neck in planning CT. (C) Image of the upper neck in cone-beam CT. (D) Typical fusion image with planning CT. The involved lymph nodes are outlined in red. CT, computed tomography; NPC, nasopharyngeal carcinoma.
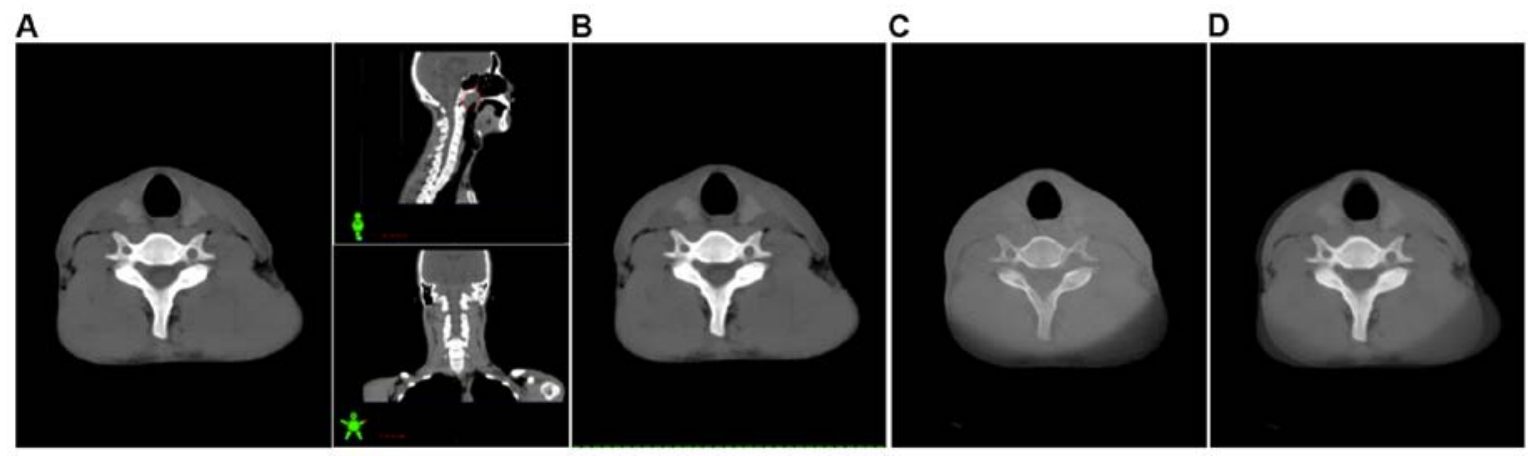

Figure 3. Image registration in the lower neck region by automatic bone matching and manual fine-tuning. (A) Planning CT images of the cross section, coronal plane and sagittal plane in a patient with NPC; cervical vertebrae 4-6 were used as a bony landmark for automatic bone matching and manual fine-tuning method. (B) Image of the lower neck in planning CT. (C) Image of the lower neck in cone-beam CT. (D) Typical fusion image with planning CT. The involved lymph nodes are outlined in red. CT, computed tomography; NPC, nasopharyngeal carcinoma.

Setup errors of the different levels following ANOVA. To establish if there were any statistical differences amongst the measured setup errors in the various regions, bony landmarks were used to represent the different regions: The nasal septum and pterygoid process represented the head; the upper neck was represented by cervical vertebrae 1-3; and the lower neck was denoted by cervical vertebrae 4-6. One-way ANOVA was performed to compare the setup errors in the different levels (upper neck, lower neck and head) and different directions (RL, SI and AP). Significant differences were observed among the different directions and the different levels (Table VI). The results demonstrated that the setup errors in all of the directions were significantly different when comparing the NPC levels of interest $(\mathrm{P}<0.01)$. Within each direction, the RL and AP directions had the greatest number of setup errors in the lower neck region, while the SI direction had the greatest number of setup errors in the upper neck region.

ROC curves. To evaluate the sensitivity and specificity of the CBCT examinations, ROC curve analysis of each 

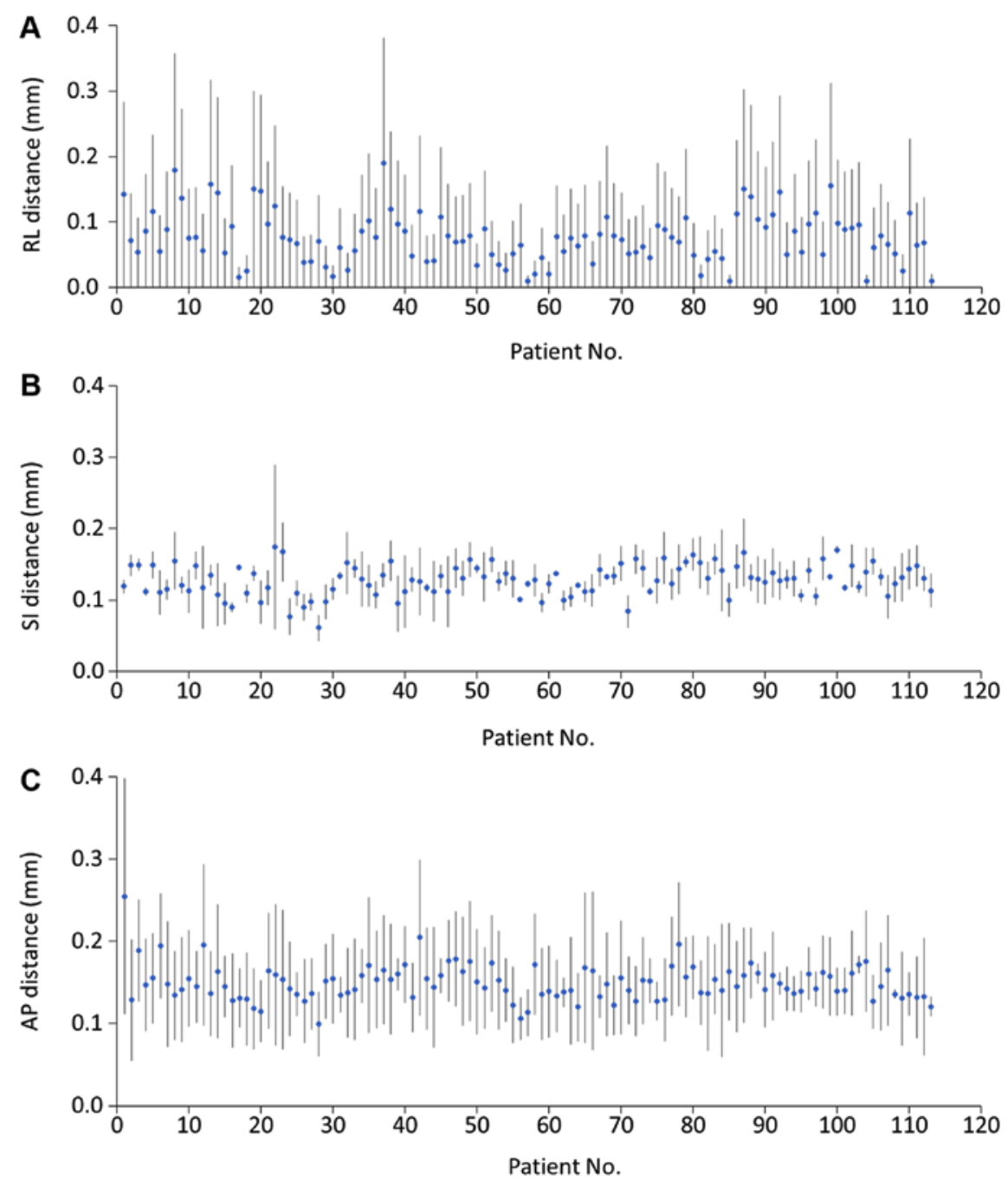

Figure 4. Distribution of setup errors amongst participants. Setup errors of 113 patients in the (A) RL, (B) SI and (C) AP direction. Data are presented as the mean \pm standard deviation. RL, right-left; SI, superior-inferior, AP, anterior-posterior.

direction and overall ROC analysis of the three directions were performed. The overall ROC analysis was comprised of all of the data, regardless of the direction. These values assisted in describing the abilities of the CBCT to correctly give a value where a setup error existed and to correctly rule out the absence of a setup shift depending on the set shift cut-off values. To achieve this goal, individual systematic errors were compared with the calculated MPTV values. The essential part of the curve is the area under the curve. For the AP, SI and RL directions respectively, the ROC analysis revealed perfect sensitivity in each direction (Fig. 5A-C). For the combined ROC curve analysis, there was an area of 0.709 , which indicates fair levels of accuracy (Fig. 5D). Thus, the CBCT examination was able to correct the setup errors and thereby contribute to accurate RT.

\section{Discussion}

The distribution of radiation doses of IMRT in NPC cases is solely based on the volume of data from planning CT scans; however, these images only provide the anatomical structures of the patient at that particular time without considering the daily changes in patients with regard to target volumes, OARs and the anatomic position (15). The existence of steep dose gradients between the structures may imply that lower doses reach the primary tumor and the surrounding normal tissues receive an overdose. Setup errors have a great effect on IMRT due to its sharp dose gradient. In situations where setup errors exist, a tiny deviation in the isodose shift may significantly lower the dose in the target volume and increase the doses administered to the OARs during the whole course of the IMRT treatment. When the target region receives a reduced radiation dose, this can lead to local tumor recurrence, over-irradiation of normal tissues, which causes unnecessary toxicity, and it can ultimately increase the probability of further complications. Tumor regression coupled with changes that occur in the target position and anatomical structures leads to the reduction of treatment accuracy during the total course of RT. In addition, errors can occur at the localization, planning or delivery stages of the treatment (12).

Rigid immobilization and frequent treatment portal verification form an important part of image-guided RT (IGRT). A number of institutions that offer head and neck tumor treatment employ uniform MPTVs of 3-5 mm with applicable image guidance protocols to account for setup errors during the course of the treatment (16). A comprehensive review 
A

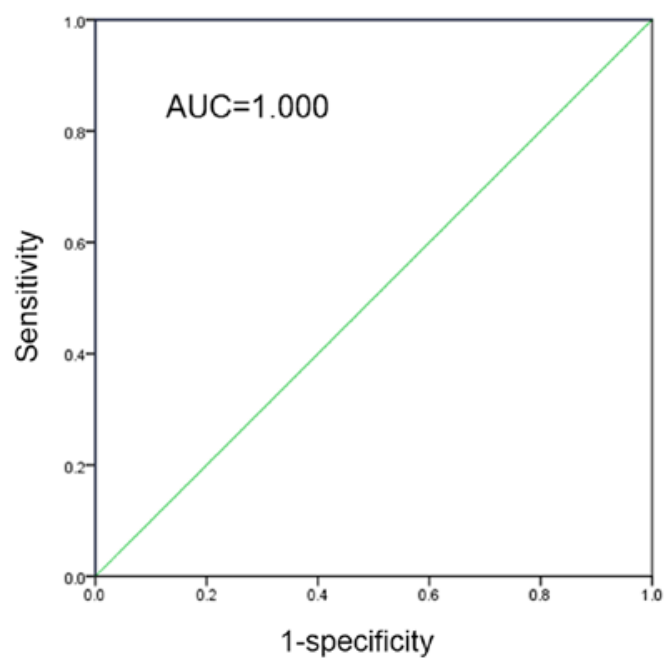

C

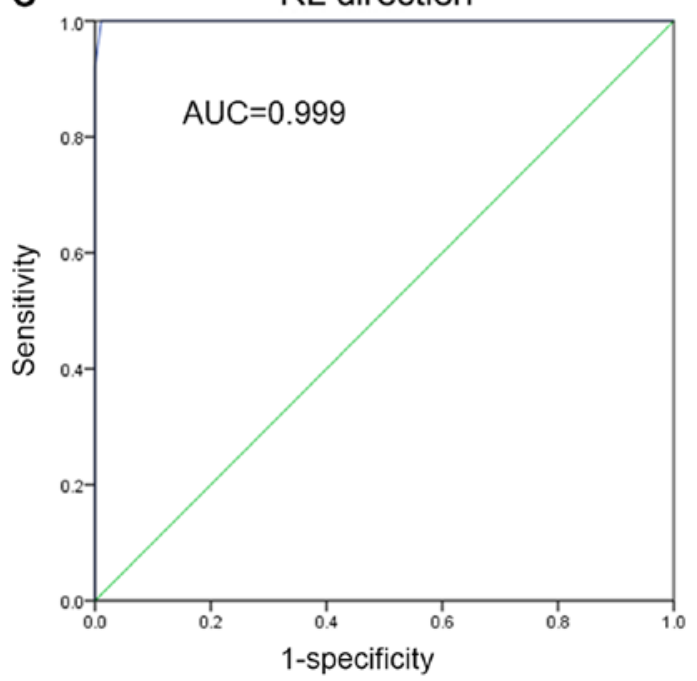

B

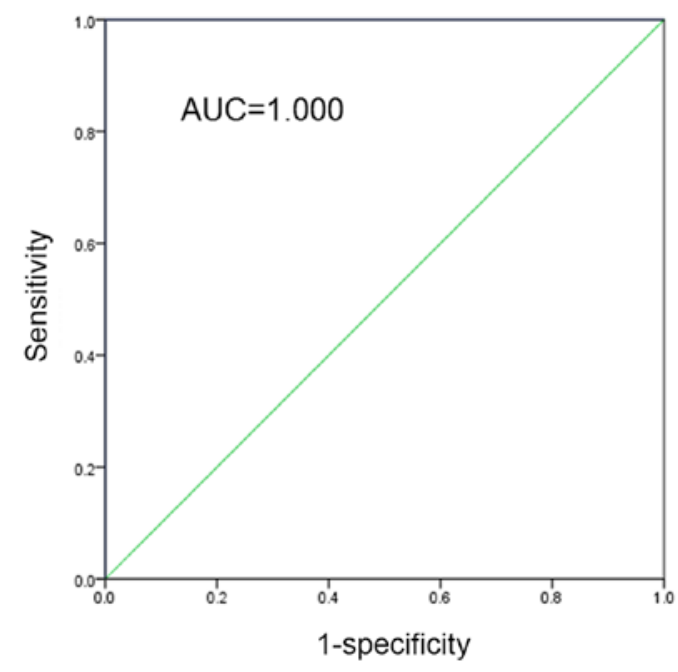

D

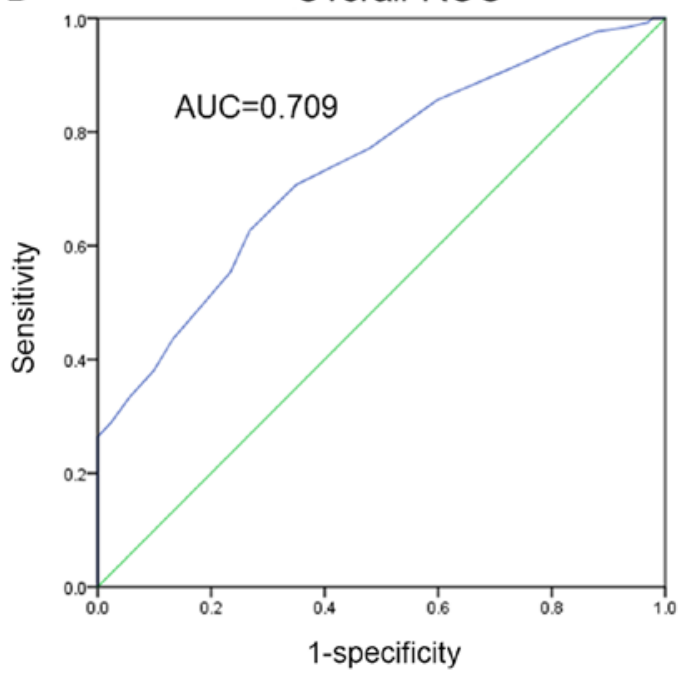

Figure 5. ROC curves of cone beam computed tomography plots. ROC curves demonstrate the area of the plots in the (A) AP direction, (B) SI direction, (C) RL direction and (D) overall plot in all directions. ROC, receiver operating characteristic; AP, anterior-posterior; SI, superior-inferior; RL, right-left; AUC, area under the curve.

Table III. Translation shifts of $>2 \mathrm{~mm}$ in RL, SI and AP directions of head, upper neck, and lower neck.

\begin{tabular}{lccc}
\hline & \multicolumn{3}{c}{ Interfraction $>2 \mathrm{~mm}, \mathrm{n}(\%)$} \\
\cline { 2 - 4 } Region & $\mathrm{RL}$ & $\mathrm{SI}$ & $\mathrm{AP}$ \\
\hline Head & $1(0.2)$ & $0(0.0)$ & $7(2.1)$ \\
Upper neck & $0(0.0)$ & $5(1.5)$ & $0(0.0)$ \\
Lower neck & $62(18.3)$ & $0(0.0)$ & $2(0.6)$ \\
\hline
\end{tabular}

RL, right-left; SI, superior-inferior, AP, anterior-posterior.

focusing on setup verification through the use of portal imaging by Hurkmans et al (17) concluded that an SD of $\leq 2 \mathrm{~mm}$ for random and systematic setup errors can be considered as 'state of the art' when using the currently available positioning equipment. Even though efficient head and neck immobilization can be achieved, a certain degree of movement at the neck and skull level still exists. Zhang et al (18) investigated the use of a CT-on-rails system and concluded that in the day to day setup error measurements at different levels, the greatest shift was recorded in the lower neck (C6 level). In their study of 14 patients, the differences amongst the levels were in the range of $2-3 \mathrm{~mm}$, suggesting that there was variability in the setup uncertainties in the different levels of the head and neck. With this in mind, it is therefore necessary to consider the relative positional variations when performing setup corrections or implementing treatment margins. In addition, a uniform margin may not be ideal at all of the levels of the head and neck treatments. This challenge in the differences in margins can be solved through the formulation of reasonable and sufficient margins or through the utilization of more advanced technology that yields reduced setup errors.

Several studies have investigated setup errors in the various sub-regions of the head and neck $(12,19,20)$. In all of these studies, planning CT images were matched with the CBCT views. In the present study, the errors were evaluated in three sites, namely the upper neck, lower neck and head. The head, 
Table IV. Summary of interfraction translational error in each dimension.

\begin{tabular}{lccc}
\hline Parameter & Right-left & Superior-inferior & Anterior-posterior \\
\hline Mean, mm & 1.494 & 1.279 & 1.898 \\
SD, mm & 0.217 & 0.215 & 0.427 \\
Minimum, mm & 0.000 & 0.000 & 0.000 \\
Maximum, mm & 9.000 & 8.000 & 14.000 \\
$\Sigma$ & 0.509 & 0.227 & 0.755 \\
$\sigma$ & 0.208 & 0.160 & 0.345 \\
MPTV, mm & 1.418 & 0.566 & 2.129 \\
\hline
\end{tabular}

SD, standard deviation; $\Sigma$, systematic setup uncertainty; $\sigma$ random setup uncertainty; MPTV, planning target volume margin.

which was represented by the nasal septum and pterygoid process, is an important region in NPC RT as there are critical structures in close proximity to the optic apparatus and brainstem. This bony reference point matching provides a close approximation of the target and OAR match. A previous study has also focused on the occiput, maxilla and mandible (21). The bony reference point was a novel approach to determine if there were any differences between the earlier studies and the present study.

The mandible produces different setup errors as it moves independently of the skull if it was employed for matching (21). The organs of interest in the present study were those associated with NPC, thus errors in the mandible were not measured. The upper neck (cervical vertebrae 1-3) region represents a region were the levels II and III neck lymph nodes are located. The C4-C6 region, which represents the lower neck, corresponds to the lower lymph node region.

Cheo et al (19) demonstrated that the setup errors were small in the head region when in comparison with the neck, and the errors were primarily located in the RL direction in the neck. In the present study, the largest setup error was noted in the lower neck AP direction.

van Kranen et al (20) also investigated the setup errors in eight different levels of the head and neck using regular CBCT scans. The results revealed that the systematic errors ranged between 1.1 and $3.4 \mathrm{~mm}$, whilst the random errors were observed to be $1.3-2.5 \mathrm{~mm}$ in 38 cases. Their results were also suggestive of a greater incidence of errors in the lateral and AP directions of the lower neck. This was in keeping with the results reported by Polat et al (22), which suggested that local setup errors were large and thus, the current PTV may not be enough to account for these setup uncertainties. In light of this, it was suggested that in order to drive correction protocols and to reduce the impact of local setup variations, multiple ROI registrations should be performed.

A study by Djordjevic et al (21) in evaluating different correction protocols revealed that the setup errors were more prevalent in the lower neck. The systematic error ranged between 0.9 and $2.3 \mathrm{~mm}$ and the random errors ranged between 1.1 and $1.6 \mathrm{~mm}$, with the maximum number of recordings observed at the C6 level.

In a study by Ove et al (23) involving the use of rail CT to confirm position verification, the lower neck was displaced anteriorly by $3.08 \pm 0.17 \mathrm{~mm}$, and no systematic lateral or craniocaudal shifts were noted. In the RL, SI and AP directions in the lower neck, the SDs of the random errors were 3.3, 2.6 and $3.9 \mathrm{~mm}$, respectively. These results illustrated that the lower neck region exceeded the planning margins. A systematic anterior displacement was observed in the lower neck and the random errors exceeded the limits. Therefore, a larger planning boundary should be employed in the neck region. Su et al (12) conducted weekly kV CBCT-guided IMRT in 30 NPC cases. Their PTV margins were 3.0, 1.3 and $2.6 \mathrm{~mm}$ in the RL, SI and AP directions, respectively. They concluded that the setup errors of the neck region were larger than those in the head during RT.

The discrepancy of the lower neck errors being larger than those in the head can be explained by the following: i) Patients with NPC with oral mucositis during the course of RT tend to have a lowered appetite, resulting in weight loss, and ultimately the neck becomes thinner; ii) as the large neck lymph shrinks during the treatment period, the diameter of the neck is reduced significantly; iii) as a result, the mask becomes loose, which then allows the neck to move laterally; and iv) during the late phases of the RT course there will be a different degree of radioactive dermatitis in the neck due to pain, and the patient will automatically move their body and shift position (12).

The setup error difference in the head and neck is of clinical importance due to the following: i) IGRT with IMRT monitors the patient's physical changes daily or weekly, if errors are detected corrections can be made early; ii) the neck region is more flexible in comparison to the head, hence it is necessary to better immobilize it in order to keep head and neck setup errors to a minimum (24); iii) detection of changes in contours due to shrinking cervical lymph nodes will allow for the modification of the ROIs and therefore alter the treatment plan in order to lower the radiation dose in the spinal cord and skin; iv) during the treatment course, active prevention and control of oral mucositis should be considered and the patients with NPC should be encouraged to eat or have enteral nutrition in order to maintain body weight; and v) radioactive dermatitis should not be overlooked (12). In cases of severe lesions, the therapy sessions can be deferred to a later stage. Head and neck motion that arises due to discomfort should be kept to a minimum. Each RT unit ought to establish the CTV-to-MPTV according to the situation. Differentiating the head and neck allows for accurate boundaries to be established for RT patients (12). 
Table V. Reported planning target volume margins from the present and previous studies.

\begin{tabular}{|c|c|c|c|c|c|}
\hline $\begin{array}{l}\text { Study } \\
\text { (Author, year) }\end{array}$ & Imaging & $\begin{array}{c}\text { Correction } \\
\text { protocol }\end{array}$ & $\begin{array}{c}\text { Margin } \\
\text { range, } \mathrm{mm}\end{array}$ & $\begin{array}{c}\text { Error } \\
\text { margins, mm }\end{array}$ & (Refs.) \\
\hline Present study & $\begin{array}{l}\text { CBCT } \\
\text { CBCT }\end{array}$ & Weekly & $\begin{array}{l}\text { Head } \\
\text { Upper neck } \\
\text { Lower neck } \\
\text { Overall }\end{array}$ & $\begin{array}{c}0.90 \\
1.40 \\
2.00 \\
1.42(\mathrm{RL}) \\
0.57(\mathrm{SI}) \\
2.13(\mathrm{AP})\end{array}$ & \\
\hline Su et al, 2015 & Orthogonal kV image & None & $\begin{array}{l}\text { Skull (clivus) } \\
\text { C3 spine } \\
\text { C6 spine }\end{array}$ & $\begin{array}{l}3.20-4.40 \\
4.40-5.50 \\
4.40-6.90\end{array}$ & $(12)$ \\
\hline Cheo et al, 2015 & CBCT & Weekly & $\begin{array}{l}\text { Skull (clivus) } \\
\text { C4 spine } \\
\text { C7 spine } \\
\text { Skull (clivus) } \\
\text { C4 spine } \\
\text { C7 spine } \\
\text { Overall }\end{array}$ & $\begin{array}{c}1.75-2.33 \\
2.61-4.33 \\
2.72-6.52 \\
0.15-1.20 \\
0.97-3.72 \\
1.20-6.08 \\
3.00(\mathrm{RL}) \\
1.30(\mathrm{SI}) \\
2.60(\mathrm{SI})\end{array}$ & (19) \\
\hline van Kranen et al, 2009 & CBCT & SAL & $\begin{array}{l}\text { Skull (Occiput) } \\
\text { C1-c3 spine } \\
\text { C5-c7 }\end{array}$ & $\begin{array}{l}4.60-7.00 \\
3.80-4.70 \\
5.40-6.00\end{array}$ & $(20)$ \\
\hline Djordjevic et al, 2014 & Orthogonal kV image & Daily & $\begin{array}{l}\text { Skull (maxilla) } \\
\text { C2 spine } \\
\text { C5 spine } \\
\text { Skull (maxilla) } \\
\text { C2 spine } \\
\text { C5 spine }\end{array}$ & $\begin{array}{c}5.20-5.90 \\
4.50(\mathrm{SI}), 6.50(\mathrm{AP}) \\
5.00-9.30 \\
4.20-5.90 \\
2.30(\mathrm{SI}), 2.60(\mathrm{AP}) \\
2.60-5.00\end{array}$ & $(21)$ \\
\hline Wang et al, 2009 & CBCT & None & Overall & $\begin{array}{c}0.70 \\
-0.70 \\
0.30\end{array}$ & $(25)$ \\
\hline Mongioj et al, 2011 & $\mathrm{CT}$ & None & Overall & $\begin{array}{l}3.40(\mathrm{RL}) \\
3.00(\mathrm{SI}) \\
3.20(\mathrm{AP})\end{array}$ & $(26)$ \\
\hline Dionisi et al, 2012 & CBCT & None & Overall & $\begin{array}{l}3.48(\mathrm{RL}) \\
4.08(\mathrm{SI}) \\
4.33(\mathrm{AP})\end{array}$ & $(27)$ \\
\hline Kapanen et al, 2013 & Orthogonal kV image & Weekly & $\begin{array}{l}\text { Skull (occiput) } \\
\text { C1-c2 spine } \\
\text { C5-c7 }\end{array}$ & $\begin{array}{c}6.10(\mathrm{SI}), 8.30(\mathrm{AP}) \\
4.80-7.00 \\
4.90-5.70\end{array}$ & (28) \\
\hline Anjanappa et al, 2017 & $\mathrm{kV}$ images & None & $\begin{array}{l}\text { Clivus } \\
\text { C3 } \\
\text { C6 }\end{array}$ & $\begin{array}{l}4.40(\mathrm{SI}), 4.00(\mathrm{AP}), 3.20(\mathrm{RL}) \\
5.50(\mathrm{SI}), 5.00(\mathrm{AP}), 4.40(\mathrm{RL}) \\
6.40(\mathrm{SI}), 4.40(\mathrm{AP}), 6.90(\mathrm{RL})\end{array}$ & $(29)$ \\
\hline
\end{tabular}

CBCT, cone beam CT; SAL, shrinking action level; AP, anterior-posterior; SI, superior-inferior; RL, right-left.

In the present study, kV CBCT-guided IMRT was utilized in 113 NPC cases. The CBCT scan images were matched to the planning CT images to establish the different setup errors in the upper neck, lower neck and head. Comparisons were then performed for the three levels, in the RL, AP and SI directions. As there are reports on the local setup error variations that exceed overall patient setup uncertainty in head and neck carcinoma, the present study performed registration at 
Table VI. Analysis of variance of setup errors in different regions in RL, SI and AP directions.

\begin{tabular}{lcccrr}
\hline Direction & Head & Upper neck & Lower neck & F-value & P-value \\
\hline RL, mm & $1.20 \pm 0.013$ & $1.30 \pm 0.009$ & $2.00 \pm 0.013$ & 187.55 & $<0.01$ \\
SI, mm & $1.14 \pm 0.007$ & $1.40 \pm 0.011$ & $1.30 \pm 0.008$ & 24.58 & $<0.01$ \\
AP, mm & $1.23 \pm 0.020$ & $1.95 \pm 0.030$ & $2.51 \pm 0.060$ & 117.22 & $<0.01$ \\
\hline
\end{tabular}

AP, anterior-posterior; SI, superior-inferior; RL, right-left.

multiple ROIs to allow for the proper evaluation of the actual PTV margins. Currently, to the best of our knowledge, there have been no studies on CBCT utilization in IMRT that have made judgments based on ROC analysis to determine the specificity of the diagnostic tool. The analysis performed in the present study demonstrates a good test, but not a definitive one.

The results of the present study revealed that a PTV margin of $1.5,0.6$ and $2.2 \mathrm{~mm}$ in the RL, SI and AP directions, respectively, is recommended for the CBCT image registrations used. For the head, and upper and lower neck the MPTVs were 0.9, 14 and $2 \mathrm{~mm}$, respectively. The differences in the error limit can be explained by the increased frequency of scans that were performed in the current study compared with previous studies. A literature search revealed that the majority of the previous studies on this topic did not include $>80$ patients, whereas the present study included data from $>100$ patients $(12,21,24)$.

Setup errors exist in the RL, SI and AP directions in NPC under IMRT in the present study. The lower neck region has higher setup errors when compared with the upper neck and head region during RT. At Nanfang hospital the recommended MPTVs are $1.5 \mathrm{~mm}$ in the RL direction, $0.6 \mathrm{~mm}$ in the SI direction and $2.2 \mathrm{~mm}$ in the AP direction for patients undergoing IMRT with weekly CBCT scans. Frequent imaging with CBCT to verify the target volume is of importance in order to maintain the accurate delivery of radiation. Derived target margins, if applied, will avoid the possibility of target under-dosage. CBCT may largely improve the accuracy of RT by minimizing the setup errors and MPTV. The ROC plot indicated that CBCT may be a fair tool for detecting setup errors in the present study.

One limitation of the present study was that it did not account for residual errors, as a second (verification) CBCT scan following repositioning was not performed. In other studies (25-29), for the prostate bed, and head and neck, the residual errors were determined by performing a second CBCT following treatment and matching it with the planning CT. Of course, this procedure will also lead to a higher dose exposure to the patient. The assessment of the residual errors will allow for calculating CTV-to-PTV expansion margins when image guidance is used, which should in general be smaller than the values obtained in the present study. As the safety margins are usually applied in three dimensions, even a small reduction can result in a considerably reduced normal tissue volume (30). The patient weight loss during the course of treatment associated with setup errors was not analyzed even though it contributes to setup errors. In addition, daily
CBCT scans were not performed for every case included in the present study due to patient economic status and other hospital resource constraints. Noise, which is a pixel variation associated with the stochastic nature of radiation is another bias. Quantifying noise will involve detecting failures in the execution of the x-ray device in this case the CBCT scanner, by doing a comparison of the values measured and baseline performance. Noise is understood to compromise the visibility of relevant structures of anatomy.

In conclusion, CBCT was demonstrated to greatly improve the accuracy of radiotherapy by minimizing the setup errors and MPTV. It was also revealed that setup errors are more prevalent in the lower neck for head and neck CBCT.

\section{Acknowledgements}

Not applicable.

\section{Funding}

The study described in this paper was substantially supported by a grant from the National Natural Science Foundation of China (grant no. 81772914; http://www.nsfc.gov.cn/) and a grant from Guangdong Provincial Department of Science and Technology (grant No. 2016A030313738; http://www.gdstc. gov.cn/).

\section{Availability of data and materials}

The datasets used and/or analyzed during the current study are available from the corresponding author on reasonable request.

\section{Authors' contributions}

KML, ZD and LZ conceived and designed the study. JL collected the data. KML and LZ analysed and interpreted the data. KML, ZD and LZ wrote the paper. JL, KML, ZD and LZ reviewed and edited the manuscript.

\section{Ethics approval and consent to participate}

All procedures performed in this study were in accordance with the ethical standards of the institutional (Nanfang Hospital Review Board) and national research committee and with the 1964 Helsinki declaration and its later amendments. The review board approved the study. Written informed consent was obtained from all individual participants included in the study. 


\section{Patient consent for publication}

Informed consent was obtained from all participants to perform the study and to publish the findings.

\section{Competing interests}

The authors declare that they have no competing interests.

\section{References}

1. Chua MLK, Wee JTS, Hui EP and Chan ATC: Nasopharyngeal carcinoma. Lancet 387: 1012-1024, 2016.

2. Lo KW, Chung GT and To KF: Deciphering the molecular genetic basis of NPC through molecular, cytogenetic, and epigenetic approaches. Semin Cancer Biol 22: 79-86, 2012.

3. Zhong H, Chen G, Lin D and Chen G: Comparison of side effects of intensity modulated radiotherapy and conventional radiotherapy in 69 cases with nasopharyngeal carcinoma. Lin Chung Er Bi Yan Hou Tou Jing Wai Ke Za Zhi 27: 462-464, 2013 (In Chinese)

4. Han C, Chen YJ, Liu A, Schultheiss TE and Wong JY: Actual dose variation of parotid glands and spinal cord for nasopharyngeal cancer patients during radiotherapy. Int J Radiat Oncol Biol Phys 70: 1256-1262, 2008.

5. Zhang X, Li M, Cao J, Luo JW, Xu GZ, Gao L, Yi J, Huang X, Xiao J, Li S and Dai J: Dosimetric variations of target volumes and organs at risk in nasopharyngeal carcinoma intensity-modulated radiotherapy. Br J Radiol 85: e506-e513, 2012.

6. Boda-Heggemann J, Lohr F, Wenz F, Flentje $M$ and Guckenberger M: kV cone-beam CT-based IGRT: A clinical review. Strahlenther Onkol 187: 284-291, 2011.

7. Chau RM, Teo PM, Kam MK, Leung SF, Cheung KY and Chan AT: Dosimetric comparison between 2-dimensional radiation therapy and intensity modulated radiation therapy in treatment of advanced T-stage nasopharyngeal carcinoma: To treat less or more in the planning organ-at-risk volume of the brainstem and spinal cord. Med Dosim 32: 263-270, 2007.

8. van Herk M, Remeijer P, Rasch C and Lebesque JV: The probability of correct target dosage: Dose-population histograms for deriving treatment margins in radiotherapy. Int $\mathbf{J}$ Radiat Oncol Biol Phys 47: 1121-1135, 2000.

9. Wilkinson JM: Geometric uncertainties in radiotherapy. $\mathrm{Br}$ J Radiol 77: 86-87, 2004.

10. Chinese committee for staging of nasopharyngeal carcinoma report on revision of the Chinese 1992 staging system for nasopharyngeal carcinoma. Radiation Oncol 2: 233-240, 2013.

11. DICOM: Scope and Field of Application. http://dicom.nema. org/medical/dicom/current/output/chtml/part01/chapter_1.html. Accessed June 12, 2017.

12. Su J, Chen W, Yang H, Hong J, Zhang Z, Yang G, Li L and Wei R: Different setup errors assessed by weekly cone-beam computed tomography on different registration in nasopharyngeal carcinoma treated with intensity-modulated radiation therapy. OncoTargets and Ther 8: 2545-2553, 2015.

13. Stroom JC and Heijmen BJ: Geometrical uncertainties, radiotherapy planning margins, and the ICRU-62 report. Radiother Oncol 64: 75-83, 2002.

14. Zweig MH and Campbell G: Receiver-operating characteristic (ROC) plots: A fundamental evaluation tool in clinical medicine. Clin Chem 39: 561-577, 1993.

15. Tan W, Li Y, Han G, Xu J, Wang X, Li Y and Hu D: Target volume and position variations during intensity-modulated radiotherapy for patients with nasopharyngeal carcinoma. Onco Targets Ther 6: 1719-1728, 2013
16. Chen AM, Farwell DG, Luu Q, Donald PJ, Perks J and Purdy JA Evaluation of the planning target volume in the treatment of head and neck cancer with intensity-modulated radiotherapy: What is the appropriate expansion margin in the setting of daily image guidance? Int J Radiat Oncol Biol Phys 81: 943-949, 2011.

17. Hurkmans CW, Remeijer P, Lebesque JV and Mijnheer BJ: Set-up verification using portal imaging; review of current clinical practice. Radiother Oncol 58: 105-120, 2001.

18. Zhang L, Garden AS, Lo J, Ang KK, Ahamad A, Morrison WH, Rosenthal DI, Chambers MS, Zhu XR, Mohan R and Dong L: Multiple regions-of-interest analysis of setup uncertainties for head-and-neck cancer radiotherapy. Int J Radiat Oncol Biol Phys 64: 1559-1569, 2006.

19. Cheo T, Loh Y, Chen D, Lee KM and Tham I: Measuring radiotherapy setup errors at multiple neck levels in nasopharyngeal cancer (NPC): A case for differential PTV expansion. Radiother Oncol 117: 419-424, 2015.

20. van Kranen S, van Beek S, Rasch C, van Herk M and Sonke JJ Setup uncertainties of anatomical sub-regions in head-and-neck cancer patients after offline CBCT guidance. Int J Radiat Oncol Biol Phys 73: 1566-1573, 2009.

21. Djordjevic M, Sjöholm E, Tullgren O and Sorcini B: Assessment of residual setup errors for anatomical sub-structures in image-guided head-and-neck cancer radiotherapy. Acta Oncol 53: 646-653, 2014.

22. Polat B, Wilbert J, Baier K, Flentje M and Guckenberger M: Nonrigid patient setup errors in the head-and-neck region. Strahlenther Onkol 183: 506-511, 2007.

23. Ove R, Cavalieri R, Noble D and Russo SM: Variation of neck position with image-guided radiotherapy for head and neck cancer. Am J Clin Oncol 35: 1-5, 2012

24. Li H, Zhu XR, Zhang L, Dong L, Tung S, Ahamad A, Chao KS, Morrison WH, Rosenthal DI, Schwartz DL, et al: Comparison of $2 \mathrm{D}$ radiographic images and 3D cone beam computed tomography for positioning head-and-neck radiotherapy patients. Int J Radiat Oncol Biol Phys 71: 916-925, 2008.

25. Wang J, Bai S, Chen N, Xu F, Jiang X, Li Y, Xu Q, Shen Y, Zhang $\mathrm{H}$, Gong Y, et al: The clinical feasibility and effect of online cone beam computer tomography-guided intensity-modulated radiotherapy for nasopharyngeal cancer. Radiother Oncol 90: 221-227, 2009.

26. Mongioj V, Orlandi E, Palazzi M, Deponti E, Marzia F, Stucchi C, Sangalli C, Fallai C, Zonca G, Olmi P and Pignoli E: Set-up errors analyses in IMRT treatments for nasopharyngeal carcinoma to evaluate time trends, PTV and PRV margins. Acta Oncol 50: 61-71, 2011.

27. Dionisi F, Palazzi MF, Bracco F, Brambilla MG, Carbonini C, Asnaghi DD, Monti AF and Torresin A: Set-up errors and planning target volume margins in head and neck cancer radiotherapy: A clinical study of image guidance with on-line cone-beam computed tomography. Int J Clin Oncol 18: 418-427, 2013.

28. Kapanen M, Laaksomaa M, Tulijoki T, Peltola S, Wigren T, Hyodynmaa S and Kellokumpu-Lehtinen PL: Estimation of adequate setup margins and threshold for position errors requiring immediate attention in head and neck cancer radiotherapy based on 2D image guidance. Radiat Oncol 8: 212, 2013.

29. Anjanappa M, Rafi M, Bhasi S, Kumar R, Thommachan KC, Bhattacharya T and Ramadas K: Setup uncertainties and PTV margins at different anatomical levels in intensity modulated radiotherapy for nasopharyngeal cancer. Rep Pract Oncol Radiother 22: 396-401, 2017.

30. Kron T: Reduction of margins in external beam radiotherapy. J Med Phys 33: 41-42, 2008.

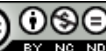

This work is licensed under a Creative Commons Attribution-NonCommercial-NoDerivatives 4.0 International (CC BY-NC-ND 4.0) License. 